\title{
Vibrations des ouvrages fixes en mer profonde
}

\author{
R. Nerzic \\ Elf-Aquitaine, Pau
}

Parmi les nouveaux concepts d'ouvrages de production pétrolière en mer profonde (300 mètres et plus), les "tours grande profondeur", structures réticulées très élancées, sont sujettes à des vibrations d'ensemble sous l'excitation des vagues.

L'étude de ces vibrations, sous excitation ambiante aléatoire et non-linéaire, a conduit au développement de nouvelles méthodes de calcul. Elles associent des techniques de réduction des degrés de liberté et des méthodes spectrales non-linéaires.

Les ouvrages de production pétrolière en mer sont installés par des profondeurs de plus en plus grandes (aujourd'hui jusqu'à $300 \mathrm{~m}$ ). Dans des profondeurs d'eau dépassant la centaine de mètres, les ouvrages fixes ont un comportement de structures rigides, ils présentent une réponse forcée à l'excitation des vagues et manifestent peu de vibrations.

Pour des profondeurs plus grandes, de nouveaux concepts ont été développés, parmi lesquels différents types de structures réticulées, regroupées sous la dénomination " tours grande profondeur ". Ces ouvrages, très élancés, sont plus souples et l'excitation des vagues conduit à des vibrations d'ensemble régies par les lois de la dynamique.

Les vibrations de ces ouvrages préoccupent les concepteurs sous deux aspects liés au dimensionnement: contraintes extrêmes lors des tempêtes, fatigue induite par les variations cycliques des contraintes pendant toute l'existence de l'ouvrage.

Leur évaluation passe par la résolution d'un problème de dynamique sous excitation ambiante, de nature aléatoire et non-linéaire, qu'il est nécessaire de bien caractériser au préalable.

\section{Structure aléatoire du champ de vagues}

Une représentation classique de la mer est le concept de houle régulière mono-directionnelle et plusieurs théories ont été développées pour la modéliser (réf. 1), la plus simple étant la théorie linéaire partant d'un profil sinusoïdal de surface libre :

$$
\eta(t)=a \sin (2 \pi f \cdot t+\varphi)
$$

$a$ : amplitude $(H / 2, H$ : hauteur crête-creux);

$f$ : fréquence $(1 / T, T=1 / \mathrm{f}$ période, $\omega=2 \pi f$ pulsation);

$\varphi$ : phase.

Cette théorie conduit à la cinématique de l'écoulement sur toute la tranche d'eau, en fonction de la profondeur $d$. A la côte $z$, la vitesse et l'accélération horizontales des particules d'eau s'écrivent:

$$
\begin{aligned}
& u(t)=\frac{\pi H}{T} \frac{\operatorname{ch}(k \cdot z)}{\operatorname{sh}(k \cdot d)} \cos (2 \pi f \cdot t+\varphi) \\
& \frac{\partial u}{\partial t}=\frac{2 \pi^{2} H}{T} \frac{\operatorname{ch}(k \cdot z)}{\operatorname{sh}(k \cdot d)} \sin (2 \pi f \cdot t+\varphi)
\end{aligned}
$$

\section{Vibrations of fixed structures in deepwater seas.}

Amongst the recent concepts of petroleum production structures in deepwater seas (300 metres and over), "the great depth towers " with very slender reticulated structures, are subjected to the entire range of vibrations under waves excitations.

The study of these vibrations, under random ambient and non-linear excitation, has resulted in the development of new calculation methodes. They bring together degrees of liberty techniques and non-linear spectral methods. 


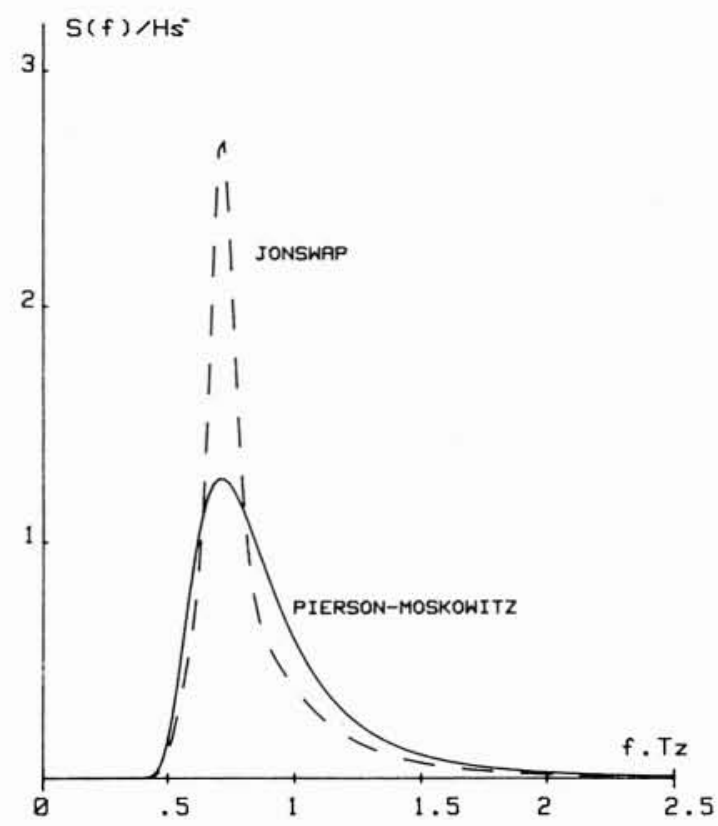

1 . Spectres de vagues.

$k$ étant le nombre d'onde $(2 \pi / L, L$ étant la longueur d'onde).

Pour caractériser de manière plus conforme à la réalité le champ de vagues, des modèles de houle irrégulière ont été développés, à partir d'hypothèses sur la surface libre (profil aléatoire gaussien), comme une superposition d'ondes élémentaires :

$$
\eta(t)=\Sigma a_{n} \cos \left(2 \pi f_{n} t+\varphi_{n}\right)
$$

On le représente alors sous forme spectrale par la densité spectrale d'énergie $S_{\eta}(f)$ de $\eta(t)$, avec la relation suivante pour la bande de fréquence à $f_{n}$ :

$$
S_{\eta}(f) \cdot \Delta f_{n}=\frac{1}{2} a_{n}^{2}
$$

$\Delta f_{n}$ étant la largeur de bande à la fréquence $f_{n}$, les phases $\varphi_{n}$ étant aléatoires et distribuées uniformément.

Cette représentation donne accès à la cinématique de l'écoulement, à partir de la théorie de houle linéaire. Par exemple, le spectre des vitesses s'écrit à partir de la relation (1.2) :

$$
S_{u}(f)=(2 \pi f)^{2} \frac{c h^{2}(k z)}{s h^{2}(k d)} \cdot S_{\eta}(f)
$$

Différents modèles de spectres de vagues sont applicables selon le type de mers (fig. 1). Ils sont caractérisés généralement par les paramètres $\mathrm{Hs}$ et $\mathrm{Tz}$ reliés aux moments d'ordre 0 et 2 du spectre:

$H s:$ hauteur significative $\left(H s=4 \sqrt{\left.m_{0}\right)}\right.$

$T z$ : période moyenne apparente $\left(T z=\sqrt{\left.m_{0} / m_{2}\right)}\right.$

On considère généralement qu'un état de mer est stationnaire sur une durée de 3 heures et cette modélisation est alors utilisée pour calculer la réponse de l'ouvrage en houle irrégulière.

Pour une description complète des conditions de mer auxquelles sera soumis l'ouvrage durant son existence (plusieurs dizaines d'années), on établit également, en fonction des conditions de site, les distributions à long terme de $H s$ et $T z$, et leur répartition par direction. Cette représentation est utilisée pour accumuler les sollicitations dans les études de fatigue.
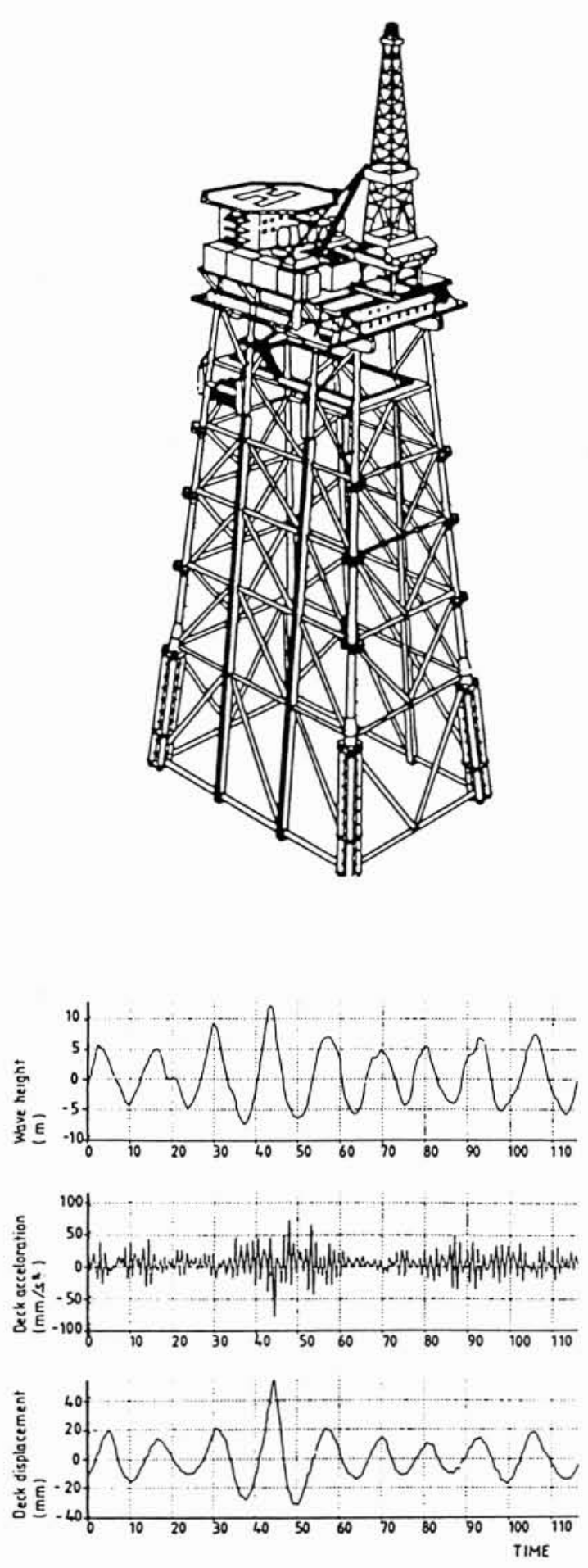

2. Plate-forme DP2 : réponse forcée aux vagues. 


\section{Forces exercées sur les ouvrages}

Les ouvrages considérés ici sont des structures réticulées constituées de treillis de barres (fig. 2). Ils sont " transparents " aux vagues (l'écoulement est considéré comme non perturbé par l'ouvrage, les dimensions des sections des barres étant faibles devant les paramètres caractéristiques de l'écoulement: hauteur de vagues et longueur d'onde), et les forces exercées par les vagues sont décrites par l'équation de Morison, qui s'écrit pour un élément de barre comme la somme d'une force de traînée et d'une force d'inertie :

- la force de trainée est proportionnelle au carré de la vitesse relative du fluide $u-\dot{x}$ ( $\dot{x}$ étant la vitesse instantanée de l'élément):

$$
F_{d}=\frac{1}{2} \rho C_{m} S|u-\dot{x}|(u-\dot{x})
$$

- la force d'inertie dépend de l'accélération relative du fluide $\dot{u}-\ddot{x}$ : on sépare la part due au fluide et celle due à l'accélération de l'ouvrage, cette dernière est le terme de masse ajoutée $m_{a} \ddot{x}$, et la force due au fluide s'écrit :

$$
F_{i}=\rho C_{d} V \dot{u}
$$

Dans ces équations :

$-S$ et $V$ représentent la surface projetée et le volume de l'élément par unité de longueur;

- $\rho$ est la masse volumique;

- $C_{d}$ et $C_{m}$ sont les coefficients de traînée et d'inertie, déterminés expérimentalement.

L'excitation des vagues présente la particularité d'être non-linéaire par le terme de traînée, un autre type de non-linéarité devant être introduit également pour l'effet des fluctuations de surface libre. D'autre part, les forces exercées sont reliées aux vitesses et accélérations relatives du fluide, ce qui indique une interaction fluidestructure.

\section{Vibrations d'ensemble des tours grande profondeur}

Pour dimensionner un ouvrage, on cherche à évaluer les contraintes induites par ses déformations, et dans le cas d'un ouvrage souple, on traite deux problèmes séparément :

- l'étude de la réponse de l'ouvrage à l'excitation des vagues;

- le passage de la déformation d'ensemble aux efforts internes, qui n'est pas abordé ici.

\section{a) Equation du mouvement}

Pour l'étude du mouvement d'ensemble, l'ouvrage est ramené à un système à $n$ degrés de liberté (ceux des nœuds de liaison des barres) et l'équation du mouvement s'écrit selon les lois de la dynamique après transposition du terme de masse ajoutée $m_{a} \ddot{x}$ au premier membre :

$$
M \ddot{X}+C \dot{X}+K X=F(t)
$$

- $M, C, K$ sont les matrices de masse, d'amortissement et de raideur du système à $n$ degrés de liberté;

- $X$ est le vecteur déplacement (degré $n$ );

- $F(t)$ est le vecteur force extérieure, appliqué à chaque degré de liberté, somme des forces $F_{d}$ et $F_{i}$ (2.1 \& 2.2).

Avant de se pencher sur la résolution de cette équation, on peut tirer des enseignements de l'étude des vibrations d'un système à 1 degré de liberté sous excitation harmonique. L'amplification dynamique est contrôlée par la fréquence de l'excitation, par la fréquence propre du système et son amortissement.

Or pour des profondeurs d'eau d'une centaine de mètres, les premières fréquences propres des ouvrages sont de l'ordre de $0,6-0,8 \mathrm{~Hz}$, éloignée de la gamme de fréquence des vagues, qui est typiquement de 0,05 à 0,2 $\mathrm{Hz}(20$ à $5 \mathrm{~s})$.

De ce fait, en négligeant en $1^{\text {ère }}$ approximation les excitations harmoniques induites par les non-linéarités, et en considérant uniquement l'amortissement structurel (2\%) l'amplification dynamique est peu marquée : on a une réponse forcée quasi-statique. Ceci a pu être vérifié expérimentalement, à partir de mesures effectuées sur un ouvrage du champ de Frigg (Elf Aquitaine Norge) : les vibrations sont peu marquées (fig. 2, réf. 2).

Par des profondeurs plus grandes, la situation est différente. L'optimisation des ouvrages conduit à des concepts plus souples. Les fréquences propres sont plus faibles, de l'ordre de $0,2 \mathrm{~Hz}$ pour les premiers modes de flexion, c'est-à-dire dans la gamme d'excitation des vagues, et on ne peut alors se contenter d'examiner la réponse forcée.

La résolution de l'équation (2.1) soulève de nombreuses difficultés, en raison des non-linéarités décrites. Une résolution numérique par simulation dans le temps, à partir d'un processus de vagues déterminé d'après le spectre, est certes possible mais elle nécessite d'importants moyens de calculs : une structure à barres de ce type comprend plusieurs centaines de nœuds de jonction et la simulation doit couvrir plusieurs milliers de pas de temps pour aboutir aux résultats escomptés (extrêmes, distribution des cycles).

De nouvelles méthodes de calcul plus performantes ont été recherchées, et les développements actuels couvrent principalement les techniques de superposition modale et de sous-structuration dynamique et les méthodes spectrales non-linéaire, techniques qui peuvent être combinées pour les applications.

\section{b) Superposition modale et sous-structuration}

La superposition modale (réf. 3) consiste à rechercher les modes propres de l'ouvrage jusqu'à un ordre élevé, puis à décomposer l'équation du mouvement sur la base orthogonale des modes propres.

Pour le système non amorti, et à partir de l'équation (3.1), les déformées modales $\phi_{i}$ et les valeurs propres $\lambda_{i}$ vérifient

$$
\lambda_{i} M \phi_{i}=K \phi_{i}
$$

Si $\Lambda$ est la matrice diagonale des valeurs propres et $\Phi$ la matrice des déformés modales, on a les relations 


$$
\begin{aligned}
& \text { 'Ф } M \Phi=I \\
& ' \Phi K \Phi=\Lambda
\end{aligned}
$$

Et on peut alors décomposer $X$ sur la base modale orthogonale, tronquée au mode $r$ et avec un terme correctif :

$$
X=\sum_{i=1}^{r} \phi_{i} y_{i}+X_{c}
$$

A l'aide d'hypothèses sur la matrice d'amortissement $C$, l'équation (3.1) se décompose alors en $r$ équations :

$$
\ddot{y}_{i}+c_{i} \dot{y}_{i}+\lambda_{i} y_{i}={ }^{t} \phi_{i} F(t)
$$

dont la résolution fournit une solution approchée:

$$
\bar{X}=\sum_{i=1}^{r} \phi_{i} y_{i}
$$

la correction dite " mode-accélération " conduisant à la solution :

$$
X=K^{-1}[F(t)-(M \ddot{\bar{X}}+\dot{\bar{X}})]
$$

L'allure des déformées étant connue pour chacun des modes, cette procédure réduit le problème à résoudre à $r$ équations plus un problème de chargement pseudostatique.

La sous-structuration dynamique est une technique de condensation permettant de réduire le nombre de degrés de liberté du problème. L'idée fondamentale (réf. 4) consiste à traiter la structure comme un assemblage de sous-structures reliées entre elles par un nombre fini de liens qui imposent des contraintes à chacune des sousstructures. Le problème peut alors être séparé en deux : un problème global dans lequel n'interviennent que les nœuds de liaison, et pour chaque sous-structure un problème interne prenant en compte les contraintes à chacun des liens. Les nœuds de liaison étant choisis (nœuds des jambes principales), en indiçant $e$ leurs degrés de liberté et $i$ les degrés de liberté internes, l'équation (3.1) devient :

$$
\begin{aligned}
{\left[\frac{M_{e} M_{e i}}{M_{i e} M_{i}}\right]\left[\frac{\ddot{X}_{e}}{\ddot{X}_{i}}\right]+} & {\left[\frac{C_{e} C_{e i}}{C_{i e} C_{i}}\right]\left[\frac{\dot{X}_{e}}{\dot{X}_{i}}\right] } \\
& +\left[\frac{K_{e} K_{e i}}{K_{i e} K_{i}}\right]\left[\frac{X_{e}}{X_{i}}\right]=\left[\frac{F_{e}}{F_{i}}\right]
\end{aligned}
$$

Et à l'aide de transformations matricielles nécessitant certaines approximations, le problème global s'écrit sous la forme classique de la dynamique, mais avec un nombre de degrés de liberté fortement réduits :

$$
\bar{M}_{e} \ddot{\bar{X}}_{e}+\bar{C}_{e} \dot{\bar{X}}_{e}+\bar{K}_{e} \bar{X}_{e}=\bar{F}_{e}
$$

- les matrices $\bar{M}_{e}, \bar{C}_{e}, \bar{K}_{e}$ prenant en compte les liaisons entre les nœuds d'interface dans chaque sous-structure et entre sous-structures,

- et le vecteur $\bar{F}_{e}$ étant obtenu par un assemblage similaire de vecteurs élémentaires.

\section{c) Méthodes spectrales non-linéaires}

L'utilisation de ces techniques permet une résolution aisée du problème dans le cas d'une force extérieure linéaire. Lorsqu'elle est valide, la linéarisation de la force de traînée (éq. 2.1) conduit à une expression du type:

$$
F_{d}=k_{d}(u-\dot{x})
$$

Après passage du terme en $\dot{x}$ au premier membre, le spectre des forces $S_{F}(f)$ s'écrit localement, à partir du spectre de vagues $S_{\eta}(f)$, et avec $F_{i}=k_{i} \ddot{u}$ (éq. 2.2) :

$$
S_{F}(f)=\left[k_{d}(2 \pi f)^{2}+k_{i}(2 \pi f)^{4}\right] \frac{c h^{2}(k z)}{s h^{2}(k d)} \cdot S_{\eta}(f)
$$

La résolution des équations (3.9) sous excitation harmonique, pour l'ensemble des fréquences composant les spectres, permet de construire la fonction de transfert entre les forces et les déplacements, et de calculer ensuite le spectre de réponse.

Cependant, cette linéarisation n'est pas toujours licite. Le calcul de la réponse d'une tour grande profondeur ( fig. 3 , réf. 5 ), sous l'excitation d'un train de vagues de périodes $16 \mathrm{~s}$, montre une mise en vibration du premier mode de période propre $5,4 \mathrm{~s}$, ceci du fait que les non-linéarités des forces excitatrices induisent une excitation au $3^{\mathrm{e}}$ harmonique de la fréquence des vagues qui est proche de la fréquence propre. Par exemple, le terme de traînée en $|\sin (\omega t)| \cdot \sin (\omega t)$, fonction impaire de $t$, se développe selon $\sin (\omega t), \sin (3 \omega t) \ldots$

Des considérations de ce type ont conduit à développer des méthodes spectrales non-linéaires, afin de prendre en compte les non-linéarités de traînée et de surface libre, comme par exemple la méthode de Volterra où les non-linéarités sont écrites sous la forme d'une somme de systèmes homogènes de degrés $n$, que l'on limite dans la pratique à l'ordre 3 . Une application de cette méthode (réf. 6) au cas d'un tube présentant une fréquence propre de $0,41 \mathrm{~Hz}$ a conduit à des résultats très prometteurs comme on le voit sur les comparaisons entre spectres de réponse calculés et mesurés (fig. 4).

\section{d) Application aux "tours grande profondeur"}

Des exemples de comportement de tours grande profondeur lors d'essais en bassin ( fig. 5 , réf. 7 ) ou en mer réelle sous forte tempête ( fig. 6 , réf. 8 ) montrent que leur réponse à l'excitation des vagues est essentiellement une réponse forcée et qu'elle est bien prédite par les calculs: il s'agit dans les deux cas d'une simulation numérique en houle aléatoire utilisant les techniques de réduction du nombre de degrés de liberté mentionnées, les résultats des essais (fig. 5) étant analysés sous la forme de spectres de réponse.

3. Tour grande profondeur: mode de vibration à $5,4 s$ et vibration sous excitation harmonique à $16 \mathrm{~s}$.

4. Comparaison entre spectre de réponse mesuré et spectre calculé selon la méthode de Volterra.

5. Tour ROSEAU: spectres de réponse calculé et expérimental (essais en bassin).

6. Tour LENA : comparaison entre réponses mesurées et calculées. 


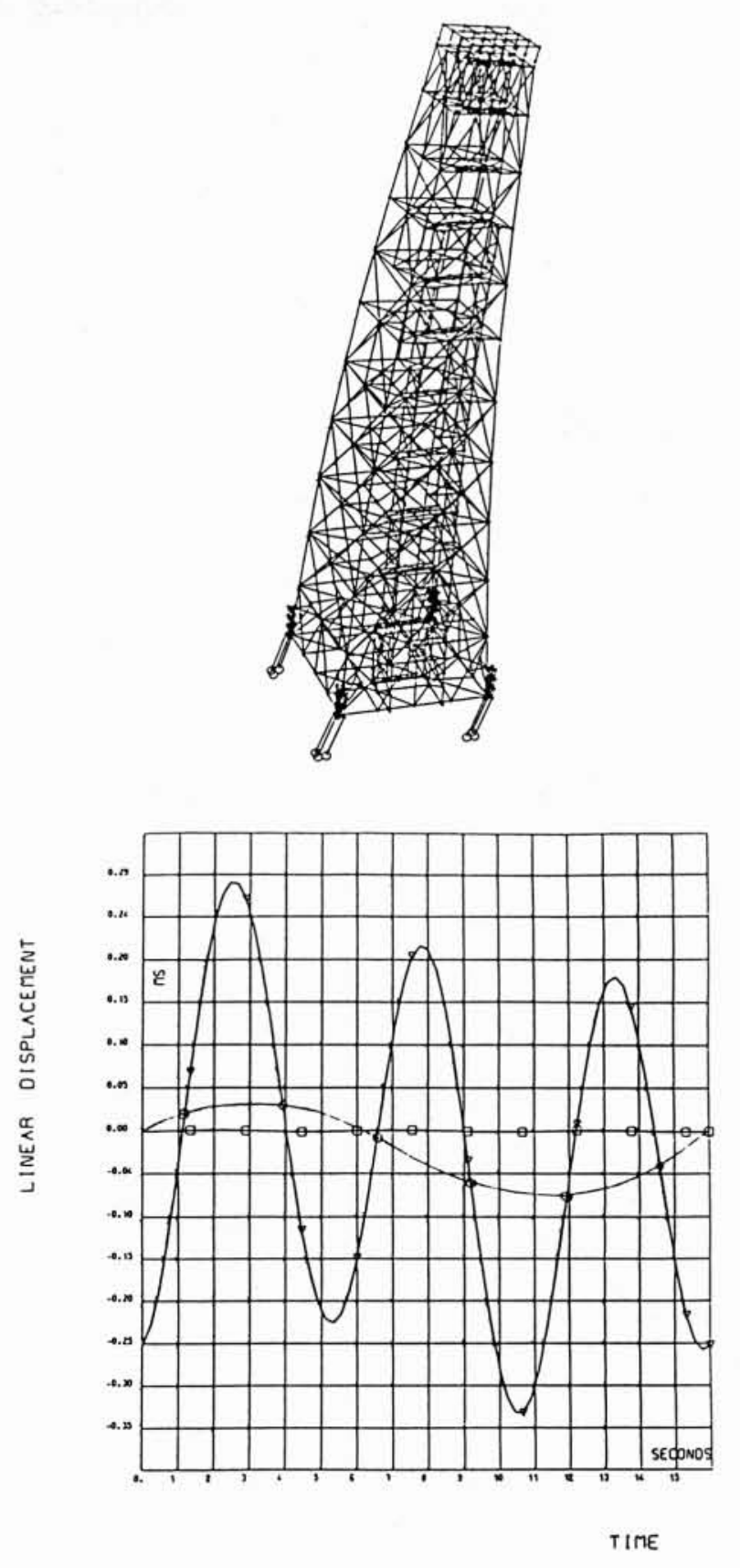

- STRAIN -
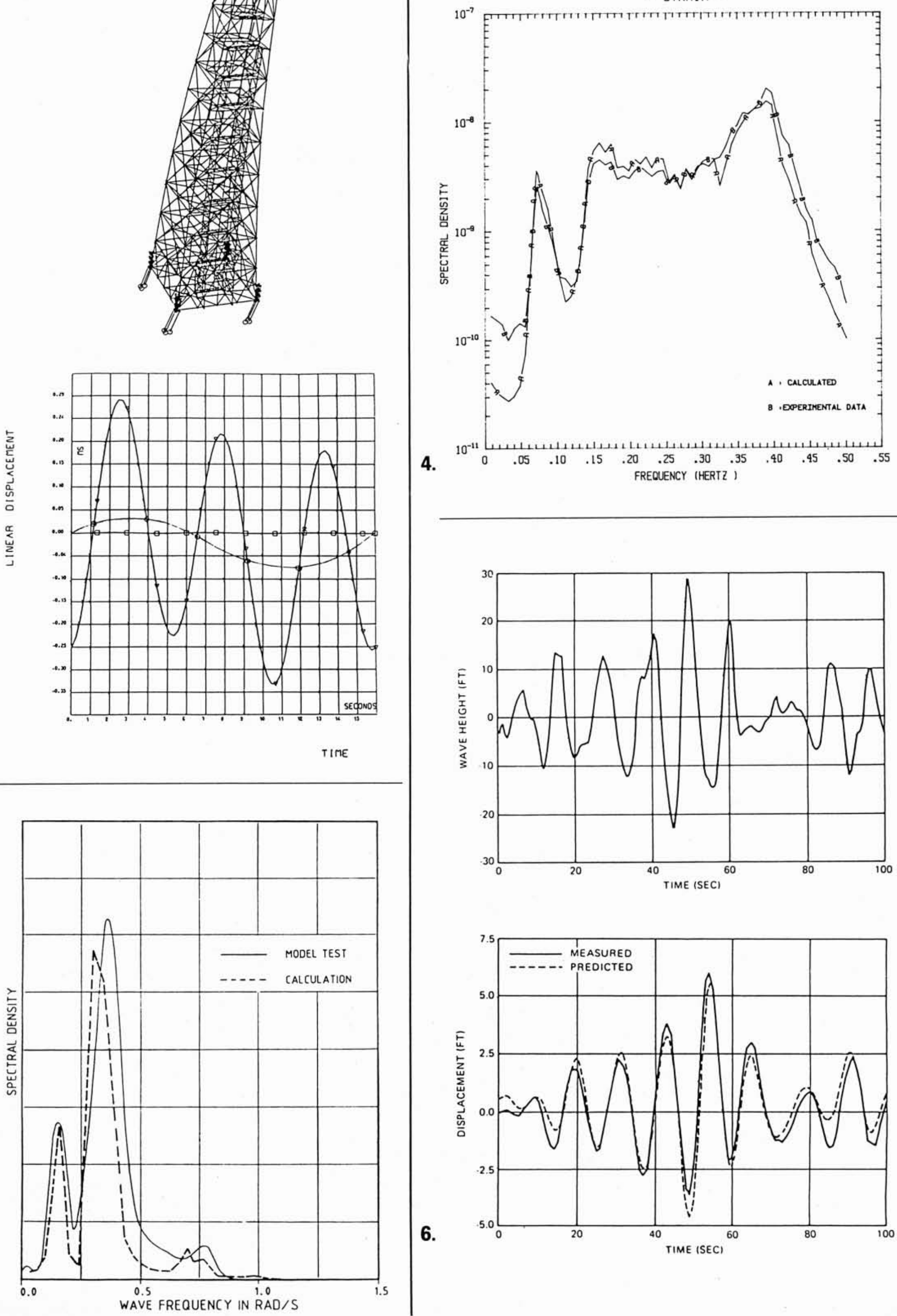

6.

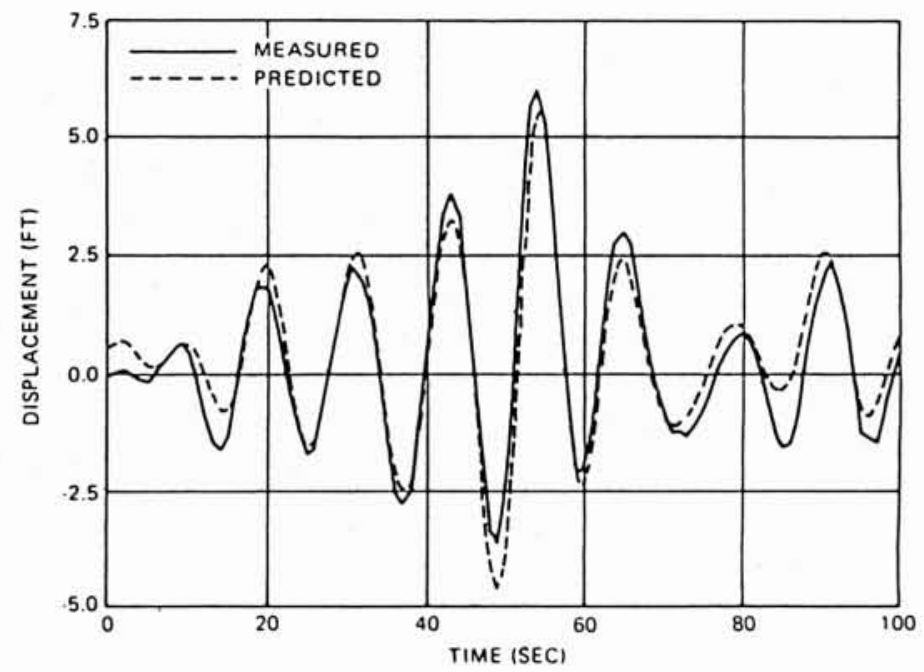




\section{PARAMETER $10000.0 \mathrm{X}$}

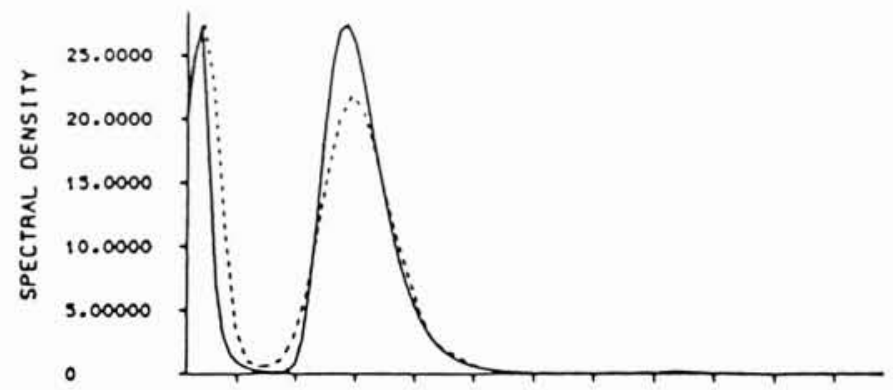

\section{‡ి}

( hZ) DOTTEO CURVE. TITE DOTAIN ANALYSIS.

\section{PARAMETER $21.1003 . F \times$}

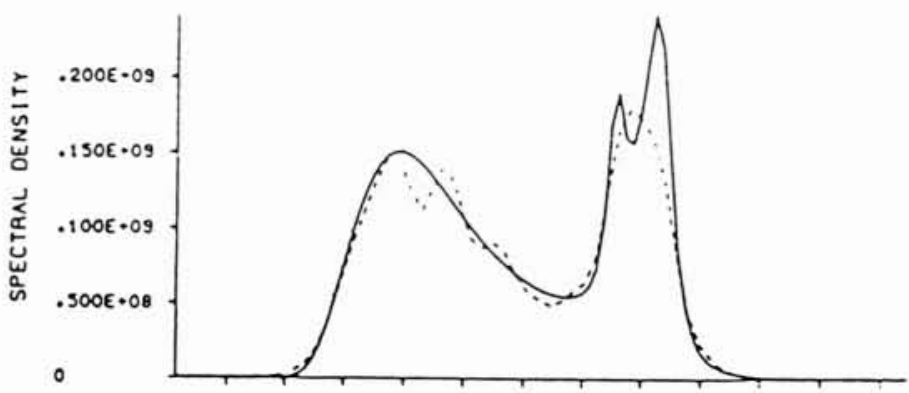

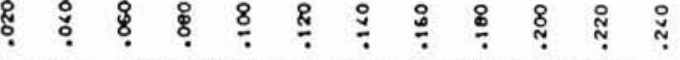 ( hz) ootteo curve, time domain analysis.}

7. Tour GAMMA: spectres de réponse calculés par méthode spectrale non-linéaire et par simulation temporelle.

Si les vibrations sont ici peu marquées, c'est d'une part parce que l'art du concepteur est de les réduire au minimum, mais aussi parce que les vibrations se présentent différemment selon les paramètres de réponse considérés comme on le constate sur la figure 7 (réf. 9).

Le spectre de déplacement en tête ( $D x)$ est constitué d'une part de réponse forcée et d'une part basse fré. quence (induite également par les non-linéarités). Par contre, le spectre d'effort $(F x)$ présente une part de vibration notable autour de $0,16 \mathrm{~Hz}$. Cette figure présente des résultats obtenus à partir de simulations dans le temps et par les méthodes spectrales non-linéaires utilisant quelques approximations vis-à-vis de la méthode de Volterra: ces résultats montrent une concordance satisfaisante entre les deux méthodes.

\section{Conclusion}

On a décrit les origines des vibrations dynamiques des ouvrages en grande profondeur sous l'excitation aléatoire et non-linéaire des vagues, ainsi que des techniques récentes permettant de les calculer avec des temps de calculs réduits. Ĺes développements de ces techniques de calcul en France sont le fait d'instituts et de sociétés d'ingéniérie, pour les besoins de nouveaux concepts soutenus par les compagnies pétrolières, et certains développements ont été possibles grâce à l'Association de Recherches sur l'Action des Eléments (ARAE), qui regroupe ces organismes.

On notera également que d'autres types de vibrations peuvent être mis en jeu sur les ouvrages en mer: vibrations consécutives à l'impact des vagues sur la structure, vibrations induites par le décollement de tourbillons sur les barres élancées, etc.

\section{Références}

[1] Vagues et ouvrages pétrolier en mer. G. SusBielles, Ch. BRATU - Editions Technip, 1981.

[2] In-service response analysis of two fixed offshore platforms J. Thébault, L. Robberstad, I. Langen, G. Agnello, Y. DOUCET, R. NERZIC - BOSS'85 - Delft.

[3] Nouvelles méthodes pour le calcul dynamique des structures souples. Rapport ARAE, 1984.

[4] Sous-structuration dynamique, validation d'une méthode de correction statique. Rapport ARAE, 1986.

[5] Jacket $350 m$ - Golfe de Guinée. Etude SNEA (P) - BOS 1987.

[6] Non-linear spectral computation of the dynamic response of a single cylinder. M. Olagnon, M. Prevosto, P. Joubert OMAE, Houston 87 .

[7] ROSEAU : a deepwater compliant platform. B. ANDRIER, Y. Delepine, J. Gauvrit - OTC, Houston 1986.

[8] Performance of the LENA guyed tower - K.M. STEELE OTC, Houston 1986

[9] Comparaison des résultats des analyses dynamiques fré quentielles et temporelles d'une plate-forme pour grande profondeur. G. DELEUIL - séminaire CLAROM, Pau 1986.

Adresse de l'auteur

Monsieur R. Nerzic

Sté nationale Elf-Aquitaine

64018 Pau Cedex

Tél. : 33.59.83.40 
M. le Président. - Sur les grandes structures de ce genre, est-ce que le fait que l'excitation n'arrive pas au même moment partout joue un rôle?

M. NERZIC. - Effectivement, on en tient d'ailleurs compte lors du calcul des ouvrages dont les dimensions dans le plan horizontal sont de l'ordre de 50 mètres. Les vagues, quant à elles, ont des longueurs d'onde d'environ 200 à 400 mètres et peuvent dépasser 25 mètres de hauteur.

M. THIRRIOT. - Est-ce que des problèmes similaires se posent sur les grosses structures en béton, comme il en existe en Mer du Nord, ou est-ce que l'inertie de ces structures supprime le risque?

M. NERZIC. - Dans ce cas, les forces d'inertie des vagues deviennent prépondérantes et les structures sont très rigides. On n'a jamais constaté ce phénomène de vibrations. Il faut dire aussi qu'il n'y a pas de structures en béton installées à des profondeurs supérieures à 100 ou 150 mètres.

M. CAILLOT. - Comment prenez-vous en compte les déplacements - qui semblent très importants - à la base de votre structure complexe? Est-ce que vous vous fondez sur des mesures et sur la déformation modale?

M. NERZIC. - Les amplitudes de déplacements à la base sont très faibles !

M. CAILLOT. - Est-ce du même ordre de grandeur que sur le sommet?

M. NERZIC. - Non. Sur une structure souple, les déplace. ments en tête sont de l'ordre du mètre, et à la base de quelques centimètres.

M. CAILLOT. - Sur une structure très haute, il y a un effet de bascule; tout est lié à ce qui se passe dans le sol.

M. NERZIC. - Au niveau de l'ancrage dans le sol, tout se fait par l'intermédiaire de pieux battus qui descendent très profondément dans le sol. Pour calculer les modes propres, on utilise une matrice de raideur approchée pour modéliser l'ancrage. Les déplacements ont été amplifiés sur la représentation graphique pour que cela soit visible à l'œil.

M. CAILLOT. - Il me semble que cette interface a une grande influence sur le résultat, comment procédez-vous?

M. NERZIC. - On découpe le sol en tranches, puis on se sert de lois donnant les relations entre forces et déformations. Pour rester dans l'hypothèse des modes propres, on est obligé de linéariser.

M. SAGNER. - Pour ce qui est du caractère non-linéaire de la traînée, vous êtes satisfaits des résultats obtenus avec l'approche de Morison? Je pense, pour ma part, qu'elle représente assez mal l'effort instantané et que, comparée à une approche linéaire, elle ne réduit pas de façon significative l'écart entre le modèle et la mesure.

M. NERzic. - Le modèle de Morison nous satisfait tout à fait, comme outil de dimensionnement.

M. SAGNER. - Est-ce que les valeurs correctes pour l'harmonique de rang 3 ne s'expliquent pas par des valeurs particulières des phases dans les spectres?

M. NERZIC. - Dans notre cas, l'harmonique de rang 3 est introduite par des non-linéarités.

Pour ce qui concerne les phases, l'analyse de spectres expérimentaux ne permet pas d'établir les déphasages entre l'excitation et la réponse de ce type d'ouvrage.

M. le Président. - Il me semble que dans les courbes que vous avez montrées, le déphasage n'est pas tellement déraisonnable. En outre, vous nous avez indiqué précédemment que les déphasages spatiaux étaient pris en compte lors de l'analyse.

M. NERZIC. - Effectivement, les déphasages entre vagues et déplacements sont cohérents.

Mais c'est lorsqu'on effectue l'analyse des mesures que l'on éprouve des difficultés à retrouver des déphasages conformes. Cela vient essentiellement de ce qu'on perd de l'information par l'analyse spectrale.

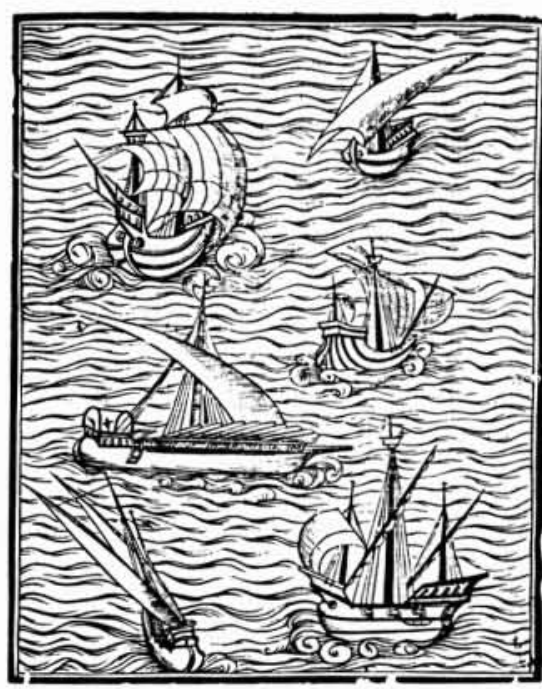

